\title{
Excited single-phase (Liquid) jets
}

\section{Other Journal Item}

\author{
Author(s): \\ Milenkovic, R.; Sigg, B.; Yadigaroglu, George
}

Publication date:

2005

Permanent link:

https://doi.org/10.3929/ethz-b-000031217

Rights / license:

In Copyright - Non-Commercial Use Permitted

Originally published in:

Journal of visualization 8(4), https://doi.org/10.1007/BF03181543 


\section{Excited Single-Phase (Liquid) Jets}

Milenkovic, R. ${ }^{1)}$, Sigg, B. ${ }^{2)}$ and Yadigaroglu, G. ${ }^{2)}$

1) Laboratorium für Thermohydraulik LTH-PSI, Paul Scherrer Institut, OVGA 415, CH-5232 Villigen PSI, Switzerland

2) Laboratorium für Kerntechnik, ETH Zentrum CLT-D3, CH-8092 Zürich, Switzerland.

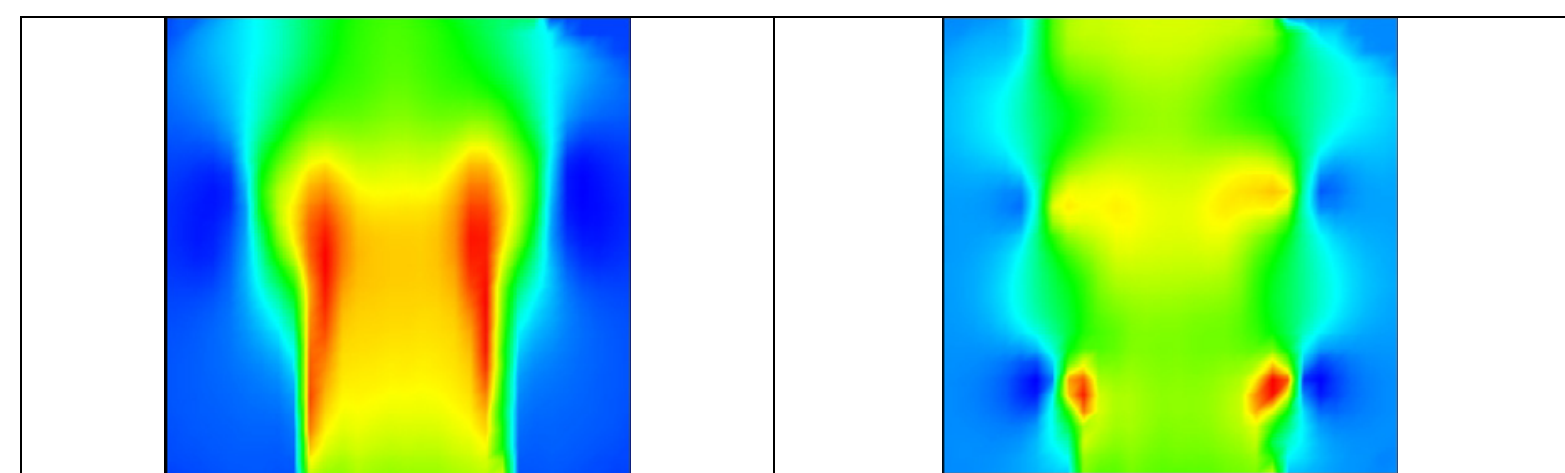

Fig. 1. Phase-averaged vertical velocity component (excitation frequency $1 \mathrm{~Hz}$-left, and $3 \mathrm{~Hz}$-right).

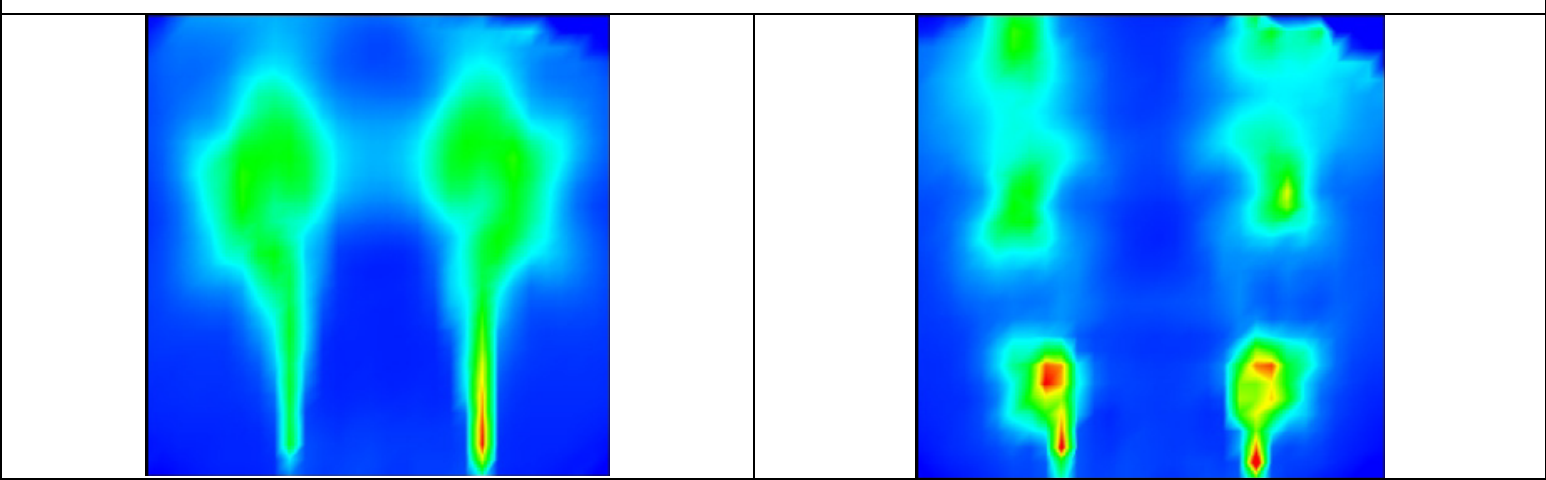

Fig. 2. Phase-averaged standard deviation of the vertical velocity component (excitation frequency $1 \mathrm{~Hz}-$ left, and $3 \mathrm{~Hz}$-right).

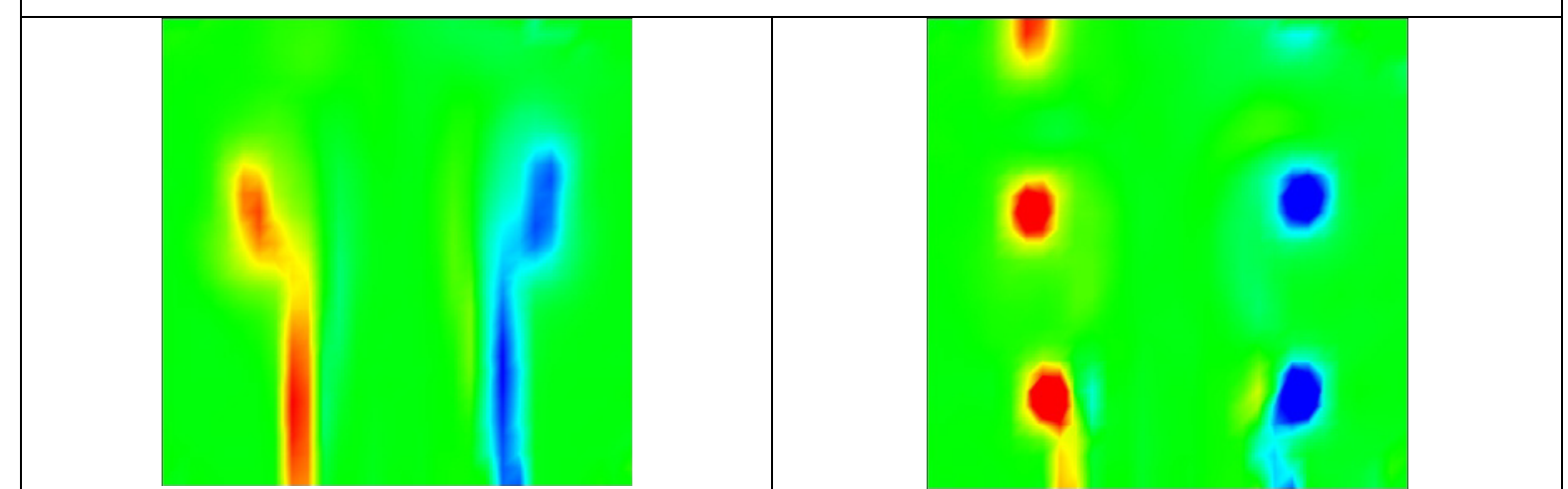

Fig. 3. Phase-averaged azimuthal vorticity component (excitation frequency $1 \mathrm{~Hz}$-left, and $3 \mathrm{~Hz}$-right)

The scalar maps show some phase-averaged quantities obtained by PIV in the developing region of a periodically triggered, axisymmetric liquid single-phase jet. Excitation frequencies are $1 \mathrm{~Hz}$ and $3 \mathrm{~Hz}$, corresponding to Strouhal number St $=0.3$ and 0.6 , respectively. Triggering of the jet shear layer leads to concentration of the shear layer vorticity in the coherent vortex rings which travel downstream at about half of the jet velocity. 